\title{
DETERMINAÇÃO E APLICAÇÃO DO COEFICIENTE DE ALARGAMENTO DE CANTONEIRAS USANDO A FÓRMULA DE EKELUND*
}

José Aparecido Pereira7

\section{Resumo}

Este trabalho descreve uma nova metodologia para análise de calibração de cantoneira bem como realizar alterações de calibrações existentes, usando para isto a fórmula de Ekelund. O ponto chave da metodologia é a transformação dos passes de cantoneira em passes planos e a determinação dos coeficientes de alargamento de cada passe. O levantamento dos fatores foi baseado em projetos existentes e em amostras coletadas em laminadores

Palavras-chave: Cantoneira; Alargamento; Ekelund; Calibração.

\section{DETERMINATION OF THE ANGLE SPREAD FACTOR BY USING THE EKELUND FORMULA}

\section{Abstract}

This paper describes a new methodology for the analysis of an angle pass design and existing projects analysis, using for this the Ekelund formula. The key point of the methodology is the transformation of the angle passes in flat ones and determining the spread coefficient of each pass. The survey of the coefficient was based on existing designs and samples collected in rolling mills.

Keywords: Angles; Spread; Ekelund; Roll Pass Design. 


\section{INTRODUÇÃO}

A fórmula de Ekelund foi desenvolvida para cálculo de alargamento em passes planos de produtos longos. Diversos softwares foram desenvolvidos para adaptar esta fórmula para canais de produtos longos (Quadrados, Redondos, Ovais, Passes de borda, entre outros) usando os retângulos equivalentes e os respectivos fatores de alargamento. Os passes de cantoneira podem ser transformados em passes planos (Chatos) através do cálculo da seção, do comprimento da fibra média e da altura média, e partir daí fazer a determinação do coeficiente de alargamento que varia em função da geometria do passe. A literatura sobre o assunto é escassa(1).

\section{MÉTODOS}

A fibra média e a altura média vão determinar o retângulo equivalente. Para determinação dos retângulos equivalentes vai ser feito o cálculo da seção e da fibra média de cada passe através do AutoCad.

\subsection{Cálculo da seção e da fibra média}

Para a determinação do retângulo equivalente são seguidos os seguintes passos:

- Levantamento do desenho do canal (Figura 1)

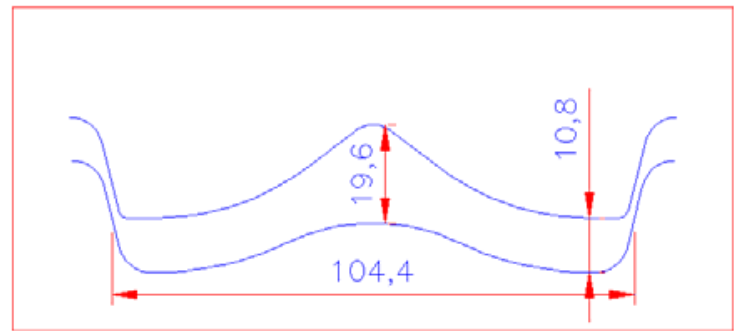

Figura 1 - Desenho do canal

- Ajuste do desenho do canal com as dimensões do passe partir de do padrão técnico ou das amostras, as dimensões devem ser consideradas a quente (Figura 2)

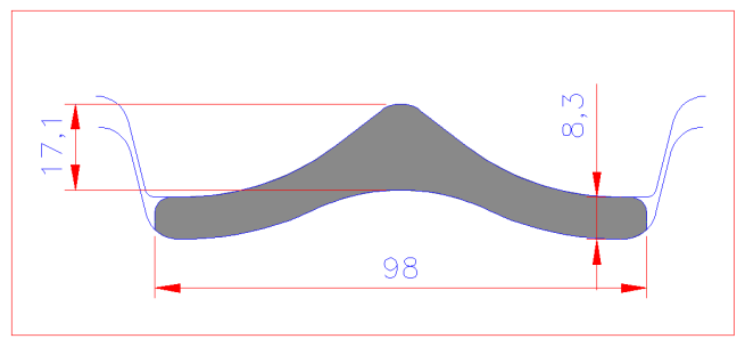

- Figura 2 - Dimensões do passe 
- Cálculo da seção e do comprimento da fibra média usando o AutoCad. A fibra média (em amarelo) representa a espinha dorsal do passe $(3)$. (Figura 3)
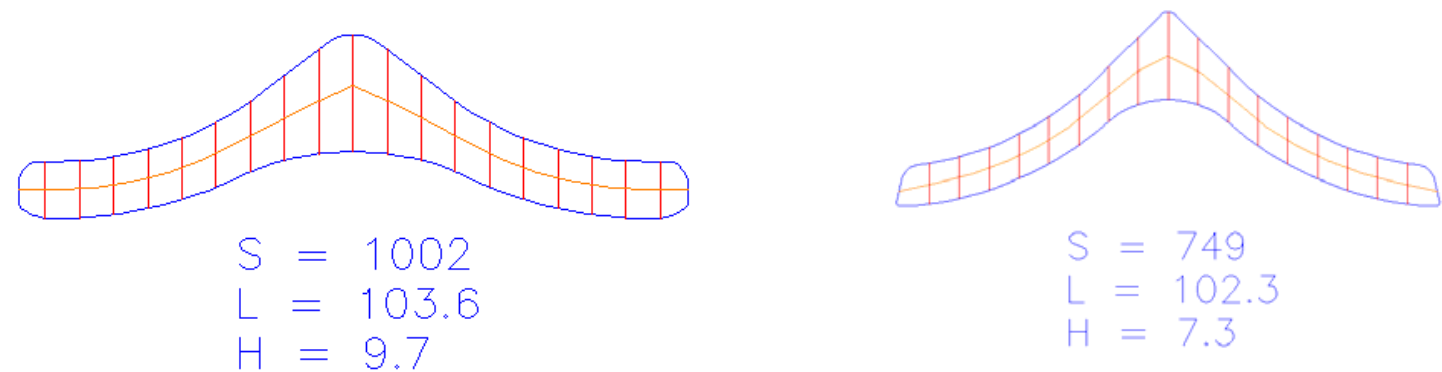

- Figura 3 - Seção, Fibra Média e Altura Média

- Dividindo-se a seção pela fibra média se acha a altura média. O Retângulo Equivalente é determinado pelo comprimento da fibra média e da altura média.

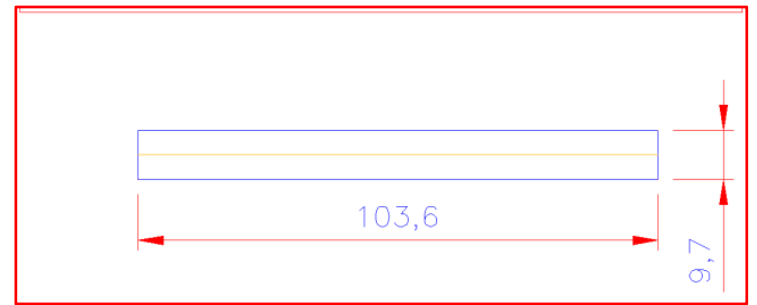

Figura 3 - Fibra Média e Altura Média (Retângulo equivalente)

\subsection{Método de calibração}

Dentre os diversos métodos de calibração de cantoneiras foi escolhido o método borboleta misto com um passe de borda intermediário partindo de um redondo com dois passes chatos e cinco passes perfilados. O passe de borda tem a finalidade de ajustar a largura dos passes além de promover um dobramento aproximando-se o ângulo de $90^{\circ}$.

A bitola escolhida é a cantoneira de $55 \times 3,5 \mathrm{~mm}$ e os dados vão ser baseados em projetos em operação e análise de amostras (Figura 4).

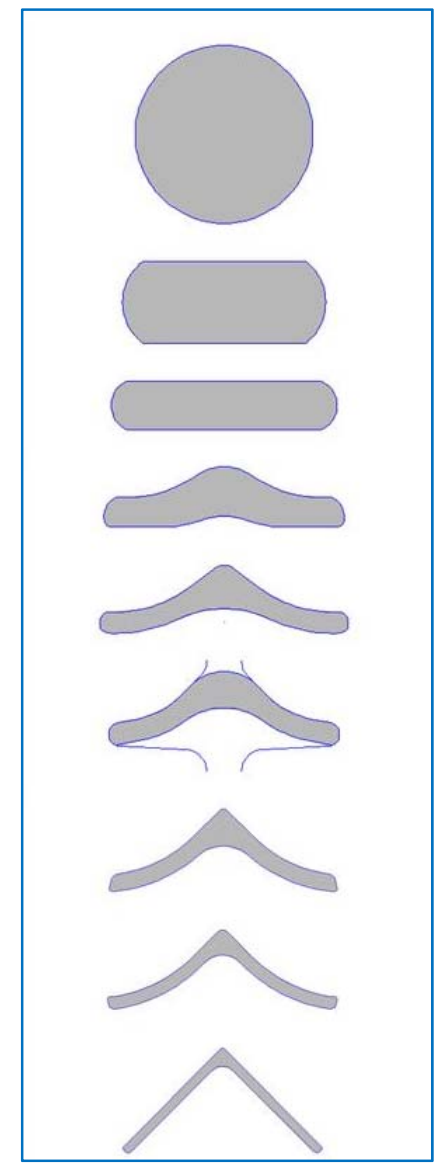




\subsection{Dimensões e dados básicos}

Na Tabela 1 são mostrados os dados básicos calculados pelos métodos acima descritos, sendo que a Fibra Média e a Altura Média definem o Retângulo Equivalente.

\section{Cantoneira 55 x 3,5mm - Dimensões Básicas}

\begin{tabular}{|c|c|c|c|c|c|c|c|}
\hline Cadeira & Formato & Espessura & Largura & Seção & Redução & Fibra Média & Alt. Média \\
\hline 10 & & 74,0 & 74,0 & 4299 & & 70,0 & 61,4 \\
\hline 11 & & 35,3 & 80,0 & 2409 & 44,0 & 80,0 & 30,1 \\
\hline 12 & & 19,0 & 89,0 & 1627 & 32,5 & 89,0 & 18,3 \\
\hline 13 & & 11,9 & 95,0 & 1352 & 16,9 & 96,3 & 14,0 \\
\hline 14 & & 8,3 & 98,0 & 1002 & 25,9 & 103,6 & 9,7 \\
\hline 15 & & 8,6 & 91,2 & 980 & 2,2 & 99,8 & 9,8 \\
\hline 16 & & 6,7 & 91,2 & 749 & 23,6 & 102,3 & 7,3 \\
\hline 17 & & 4,9 & 89,8 & 535 & 28,6 & 103,3 & 5,2 \\
\hline 18 & & 3,5 & 55,8 & 368 & 31,2 & 106,3 & 3,5 \\
\hline
\end{tabular}

Tabela 1 - Dimensões básicas

\subsection{Determinação do coeficiente de alargamento}

Como a fórmula de Ekelund tem resolução complexa (Figura 5), já que a variável B1 se encontra nos dois lados da equação, foi desenvolvida uma planilha no Excel que em conjunto com o programa Solver encontra a largura do passe laminado (B1).

$$
\begin{gathered}
B 1=\sqrt{8 \times A \times L d \times \Delta H-4 \times A \times L d \times(H 0+H 1) \times \ln \frac{B 1}{B 0}+B 0^{2}} \\
A=\frac{1.6 \times \mu \times \sqrt{R \times \Delta H}-1.2 \times \Delta H}{H 0+H 1}
\end{gathered}
$$

Figura 5 - Fórmula de Ekelund

Quando se trata de canais de passes de barras (Quadrados, redondos, ovais, chatos, etc.) são usados coeficientes de alargamento diferenciados de acordo com o formato do canal para determinação da largura da barra laminada.

Em se tratando de passes de cantoneiras esses coeficientes não são bem conhecidos, mas a análise de amostras e a literatura(1) mostram que dependendo do formato da barra de entrada e de saída eles podem variar bastante, na maioria das 
vezes causando um alargamento bem maior do que os calculados por Ekelund. Neste trabalho são mostrados os primeiros passos no estudo e determinação destes coeficientes, que pelas análises realizadas apresentam grande variabilidade e os fatores que o influenciam ainda não são bem conhecidos.

Neste caso os coeficientes vão ser determinados a partir do projeto de calibração da cantoneira $55 \times 3,5 \mathrm{~mm}$, bem como no seu padrão técnico de operação elaborado a partir da operação e análise de amostras. Os coeficientes vão ser ajustados em função da largura calculada por Ekelund e da largura do retângulo equivalente da cantoneira em análise.

A Tabela 2 mostra os dados de entrada e de saída (em azul) da planilha, sendo que para obtenção de resultado final, todos os resultados de "Delta" devem estar zerados. Na cadeira em que estiver acima de zero o Solver deverá usado sucessivamente em todas as cadeiras para se obter o resultado final.

\begin{tabular}{|c|c|c|c|c|c|c|c|c|c|}
\hline \multicolumn{10}{|c|}{ Ekelund + Solver - Dados } \\
\hline \multicolumn{10}{|c|}{ Cantoneira 55 x 3,5mm - Padrão Técnico } \\
\hline & Cad 10 & Cad 11 & Cad 12 & Cad 13 & Cad 14 & Cad 15 & Cad 16 & Cad 17 & Cad 18 \\
\hline Tipo & $\mathrm{RD}$ & $\mathrm{CH}$ & $\mathrm{CH}$ & CT & CT & $\mathrm{BC}$ & CT & CT & $\mathrm{AC}$ \\
\hline Altura (mm) & 58,1 & 30,1 & 18,3 & 15,0 & 9,7 & 99,6 & 7,3 & 5,2 & 3,5 \\
\hline Largura Calculada & 74,0 & 83,0 & 90,6 & 94,2 & 109,9 & 9,9 & 102,2 & 104,3 & 106,3 \\
\hline Seção Calculada & 4299 & 2500 & 1656 & 1412 & 1066 & 988 & 746 & 542 & 368 \\
\hline Red.Calculada & & 41,8 & 33,7 & 14,7 & 24,5 & 7,3 & 24,5 & 27,3 & 32,2 \\
\hline Diâmetro & & 385 & 385 & 365 & 385 & 385 & 385 & 385 & 385 \\
\hline Luz & & 6,6 & 16 & 3,1 & 4,9 & 12,7 & 3,6 & 3,3 & 3,5 \\
\hline Temperatura $\left({ }^{\circ}\right)$ & & 1000 & 977 & 953 & 946 & 930 & 919 & 910 & 902 \\
\hline Coeficiente Alargamento & & $100 \%$ & $100 \%$ & $280 \%$ & $280 \%$ & $70 \%$ & $165 \%$ & $150 \%$ & $140 \%$ \\
\hline Delta & & 0 & 5 & 0 & 457 & 133 & 0 & 0 & 0 \\
\hline
\end{tabular}

Tabela 2 - Entrada e saída de dados

A Figura 6 mostra a tela de resolução da cadeira 14 em conjunto com o Solver, onde se pode observar que a diferença (Delta) está maior que zero e a largura do passe é igual a $109,9 \mathrm{~mm}$. 


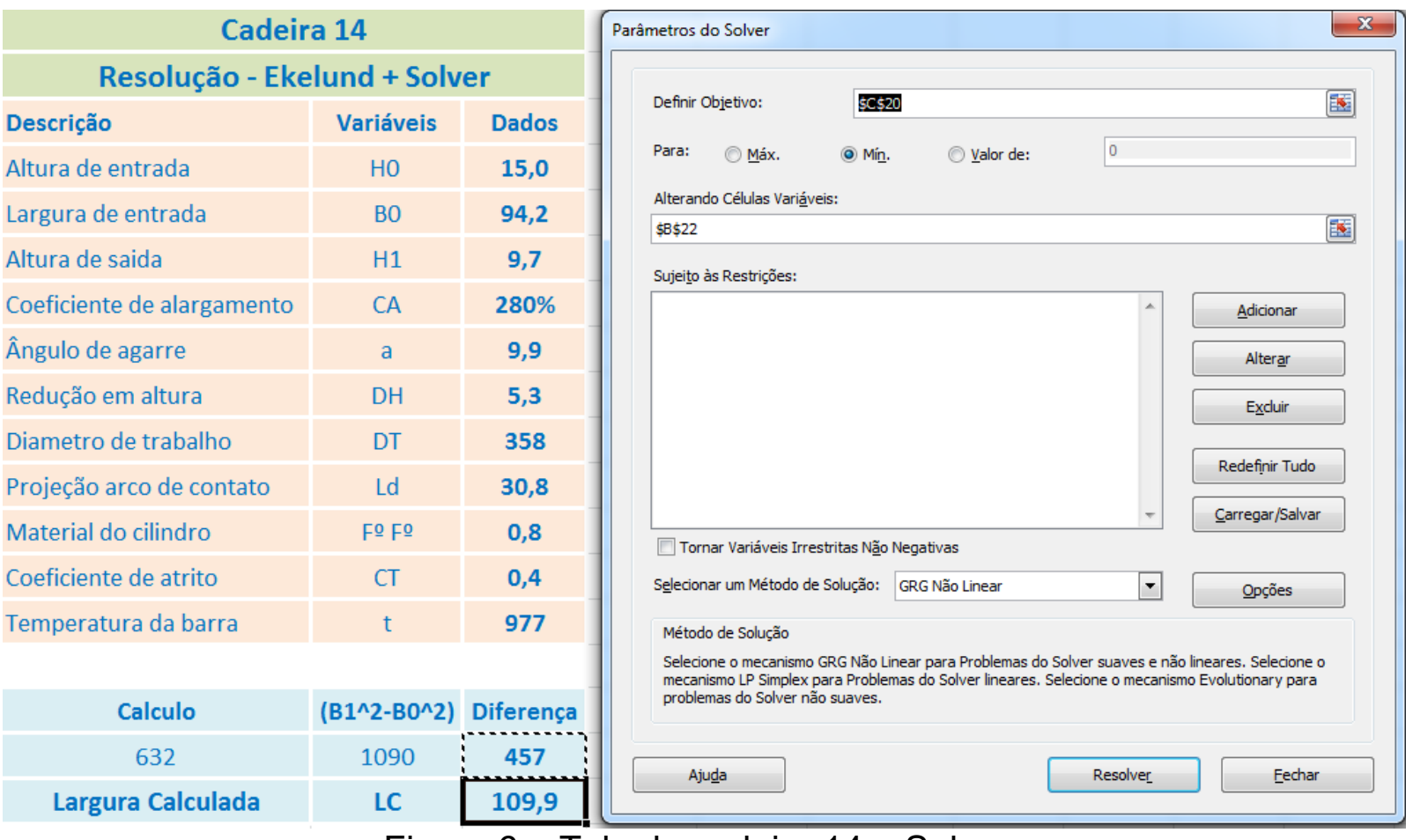

Figura 6 - Tela da cadeira $14+$ Solver

Após o acionamento do Solver a diferença vai para zero e a largura do passe passa para $103,9 \mathrm{~mm}$ (Figura 7 )

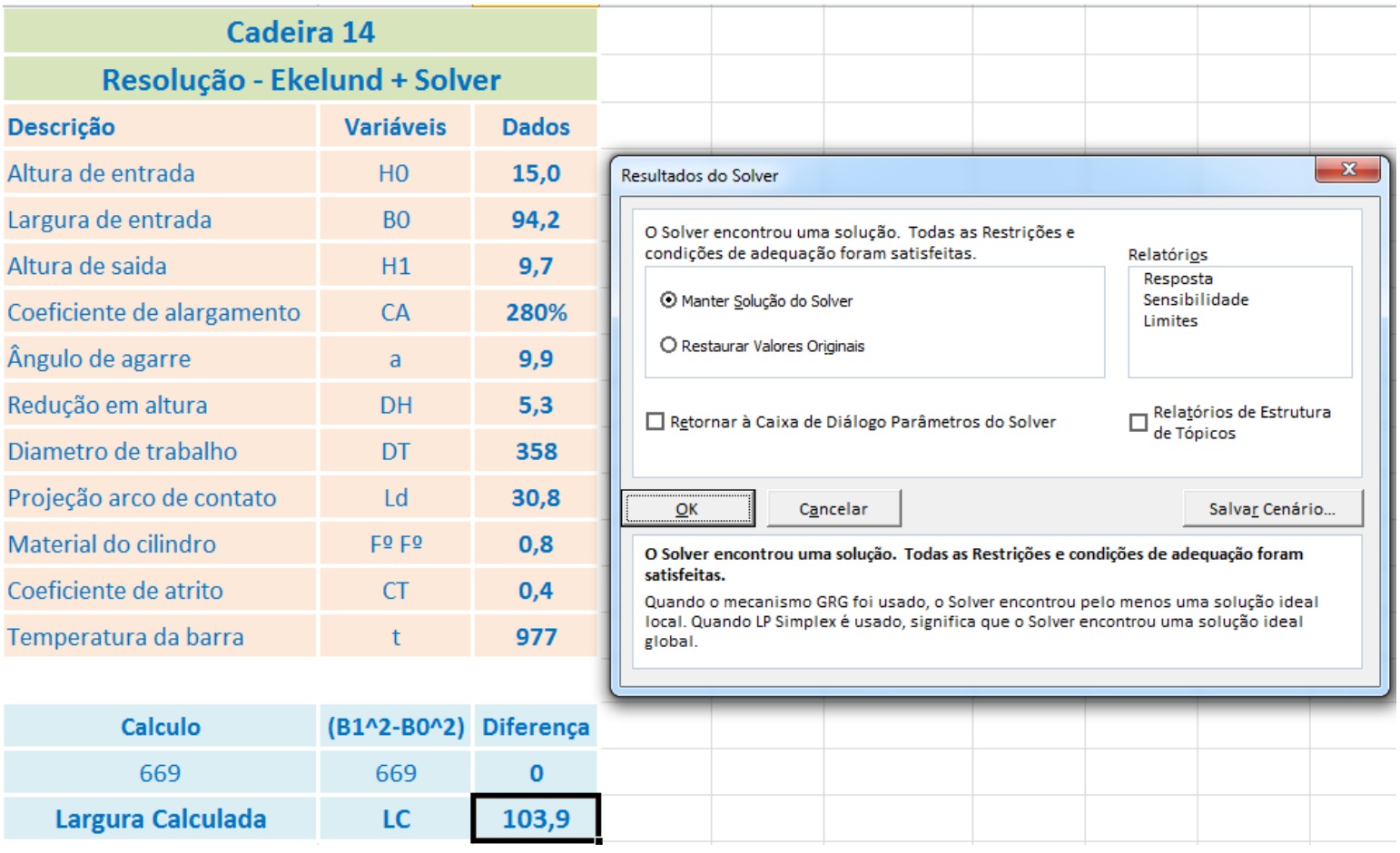

Figura 7 - Tela da cadeira $14+$ Solver

Após o acionamento do Solver em todas as cadeiras, temos o resultado final mostrando as larguras, seções, reduções e coeficientes. Mostra também a dimensão das abas no produto acabado bem como a diferença entre o passe calculado e largura do canal. 


\begin{tabular}{|c|c|c|c|c|c|c|c|c|c|}
\hline \multicolumn{10}{|c|}{ Ekelund + Solver - Dados } \\
\hline \multicolumn{10}{|c|}{ Cantoneira 55 x 3,5mm - Padrão Técnico } \\
\hline & Cad 10 & Cad 11 & Cad 12 & Cad 13 & Cad 14 & Cad 15 & Cad 16 & Cad 17 & Cad 18 \\
\hline Tipo & RD & $\mathrm{CH}$ & $\mathrm{CH}$ & CT & CT & $\mathrm{BC}$ & CT & CT & $\mathrm{AC}$ \\
\hline Altura (mm) & 58,1 & 30,1 & 18,3 & 15,0 & 9,7 & 99,6 & 7,3 & 5,2 & 3,5 \\
\hline Largura Calculada & 74,0 & 83,0 & 90,6 & 94,1 & 103,9 & 10,0 & 102,3 & 104,4 & 106,4 \\
\hline Seção Calculada & 4299 & 2500 & 1656 & 1412 & 1008 & 991 & 746 & 543 & 368 \\
\hline Red.Calculada & & 41,8 & 33,8 & 14,7 & 28,6 & 1,7 & 24,7 & 27,3 & 32,2 \\
\hline Diâmetro & & 385 & 385 & 365 & 385 & 385 & 385 & 385 & 385 \\
\hline Luz & & 6,6 & 16 & 3,1 & 4,9 & 12,7 & 3,6 & 3,3 & 3,5 \\
\hline Temperatura $\left({ }^{\circ}\right)$ & & 1000 & 977 & 953 & 946 & 930 & 919 & 910 & 902 \\
\hline Coeficiente Alargamento & & $100 \%$ & $100 \%$ & $280 \%$ & $280 \%$ & $70 \%$ & $165 \%$ & $150 \%$ & $140 \%$ \\
\hline \multirow[t]{2}{*}{ Delta } & & 0 & 0 & 0 & 0 & 0 & 0 & 0 & 0 \\
\hline & \multicolumn{2}{|c|}{ Aba a frio } & 55,0 & \multicolumn{3}{|c|}{ Diferença de abas } & 0,0 & & \\
\hline Largura do canal & & & & 96,30 & 103,60 & & 102,30 & 104,50 & 106,30 \\
\hline Diferença & & & & $-2,2$ & 0,3 & & 0,0 & $-0,1$ & 0,1 \\
\hline
\end{tabular}

Tabela 3 - Entrada e saída de dados

\subsection{Análise dos resultados do coeficiente de alargamento}

Observa-se que para esta cantoneira os coeficientes de alargamento dos passes perfilados variaram de 280 a $140 \%$. Sendo que o passe de borda ficou em $70 \%$. Neste caso os valores maiores foram nos dois primeiros passes onde a mudança de forma é mais abrupta. Neumann em Kalibrieren von Walzen (pag 169) reporta coeficientes variando de 50 a $300 \%$ dependendo do ângulo e formato do passe(2).

\subsection{Análise dos resultados de redução de seção}

$\mathrm{Na}$ Tabela 3 se pode observar que a cadeira 11 está com redução muito alta para um passe chato e que a cadeira 18 como cadeira acabadora está com redução também alta. O ideal é que as reduções nos passes de cantoneira não passem de $27 \%$ para prevenir sobrecarga no acionamento e aumentar a vida útil dos canais. $A$ cadeira 13 está com redução baixa e é a oportunidade de equalizar as reduções.

Vão ser feitas alterações nas alturas dos passes e a partir dos coeficientes de alargamento vão ser calculadas as novas larguras e identificada a necessidade ou não de alterações na largura dos canais e também checar a dimensão das abas no passe acabador..

\subsection{Alterações das reduções dos passes}

$\mathrm{Na}$ Tabela 4 são mostradas as alterações introduzidas e os resultados obtidos nas reduções (destaque verde), larguras (em azul) dos canais e dimensão da aba acabada que ficou no nominal. Observa-se que para manter o tamanho da aba acabada foi necessário abrir $1,0 \mathrm{~mm}$ na cadeira 15 . A largura dos passes mostra a necessidade de um grande aumento de largura nas cadeiras 13 e 14 (9,4 e 8,0mm) e um ajuste menor nas cadeiras 16 e $17(1,1$ e $0,7 \mathrm{~mm})$ 


\begin{tabular}{|c|c|c|c|c|c|c|c|c|c|}
\hline \multicolumn{10}{|c|}{ Ekelund + Solver - Dados } \\
\hline \multicolumn{10}{|c|}{ Cantoneira 55 x 3,5mm - Ajuste de Reduções } \\
\hline & Cad 10 & Cad 11 & Cad 12 & Cad 13 & Cad 14 & Cad 15 & Cad 16 & Cad 17 & Cad 18 \\
\hline Tipo & RD & $\mathrm{CH}$ & $\mathrm{CH}$ & CT & CT & $\mathrm{BC}$ & CT & CT & $A C$ \\
\hline Altura $(\mathrm{mm})$ & 58,1 & 35,3 & 19,0 & 12,3 & 8,5 & 100,6 & 6,5 & 4,7 & 3,5 \\
\hline Largura Calculada & 74,0 & 82,5 & 93,1 & 105,7 & 111,6 & 9,1 & 103,4 & 105,2 & 106,3 \\
\hline Seção Calculada & 4299 & 2763 & 1768 & 1300 & 949 & 916 & 672 & 494 & 368 \\
\hline Red.Calculada & & 35,7 & 36,0 & 26,5 & 27,0 & 3,5 & 26,6 & 26,5 & 25,6 \\
\hline Diâmetro & & 385 & 385 & 365 & 385 & 385 & 385 & 385 & 385 \\
\hline Luz & & 6,6 & 16 & 3,1 & 4,9 & 12,7 & 3,6 & 3,3 & 3,5 \\
\hline Temperatura $\left({ }^{\circ}\right)$ & & 1000 & 977 & 953 & 946 & 930 & 919 & 910 & 902 \\
\hline Coeficiente Alargamento & & $100 \%$ & $100 \%$ & $280 \%$ & $280 \%$ & $70 \%$ & $165 \%$ & $150 \%$ & $140 \%$ \\
\hline \multirow[t]{2}{*}{ Delta } & & 0 & 0 & 0 & 0 & 0 & 0 & 0 & 0 \\
\hline & \multicolumn{2}{|c|}{ Aba a frio } & 55,0 & \multicolumn{3}{|c|}{ Diferença de abas } & 0,0 & & \\
\hline Largura do canal & & & & 96,30 & 103,60 & & 102,30 & 104,50 & 106,30 \\
\hline Diferença & & & & 9,4 & 8,0 & & 1,1 & 0,7 & 0,0 \\
\hline
\end{tabular}

Tabela 4 - Entrada e saída de dados

\section{RESULTADOS E DISCUSSÃO}

O resultado da análise comprova a ocorrência de coeficientes de alargamento diferenciados em relação à geometria e posição dos passes. A metodologia, usando os coeficientes predeterminados, permitiu ajustar as reduções de seção levando estes valores para níveis balanceados nas diversas cadeiras do laminador. (Tabela 5)

\begin{tabular}{|c|c|c|c|c|c|c|c|}
\hline Cadeira & Formato & Espessura & Largura & Seção & Redução & Fibra Média & Alt. Média \\
\hline 10 & & 74,0 & 74,0 & 4299 & & 70,0 & 58,1 \\
\hline 11 & & 35,3 & 82,5 & 2763 & 35,7 & 82,5 & 35,3 \\
\hline 12 & & 19,0 & 93,1 & 1768 & 36,0 & 93,1 & 19,0 \\
\hline 13 & & 10,2 & 104,4 & 1300 & 26,5 & 105,7 & 12,3 \\
\hline 14 & & 7,1 & 106,0 & 949 & 27,0 & 111,6 & 8,5 \\
\hline 15 & & 7,9 & 92,0 & 916 & 3,5 & 9,1 & 100,6 \\
\hline 16 & & 5,9 & 92,3 & 672 & 26,6 & 103,4 & 6,5 \\
\hline 17 & & 4,4 & 91,7 & 494 & 26,5 & 105,2 & 4,7 \\
\hline 18 & & 3,5 & 55,8 & 368 & 25,6 & 106,3 & 3,5 \\
\hline
\end{tabular}

Tabelas 5 - Dimensões básicas - Ajustadas 


\section{CONCLUSÃO}

A metodologia utilizada com o uso do Solver mostra ser o caminho correto, nesta fase inicial, a ser trilhado para a análise, correção e desenvolvimento de Calibração de Cantoneiras. Esta metodologia, que já está sendo usada na prática, é um desenvolvimento recente e será enriquecida e ampliada a partir de mais resultados práticos que poderão ser armazenados em um banco de dados, permitindo análise com métodos estatísticos e através do desenvolvimento de um software com mais recursos e interação gráfica. Este software também será capaz de desenvolver uma nova calibração a partir de parâmetros pré-estabelecidos pelo calibrador.

\section{REFERÊNCIAS}

Horst Kuhene. Open Pass Angle Design. Institute of Roll Design Seminar. 2000.

2 Dr. Horst Neumann. Kalibrieren von Walzen. Leipzig: VEB Doutscher Verlag;1976

3 SMS. Manual - Roll Pass Design Training. Dusseldorf. Schoemann-Siemag AG;1975 